\title{
EFFECT OF NITROGEN FERTILIZATION LEVELS ON GRAIN YIELD AND YIELD COMPONENTS IN TRITICALE BASED ON AMMI AND GGE BIPLOT ANALYSIS
}

\author{
ORAL, E. \\ Department of Seed Production, Klzlltepe Vocational Training High School, Artuklu University \\ 21500 Kizlltepe, Mardin, Turkey \\ (e-mail: eroloral65@gmail.com; phone: +90-546-746-6266) \\ (Received 22 ${ }^{\text {nd }}$ May 2018; accepted $17^{\text {th }}$ Jul 2018)
}

\begin{abstract}
This study examined the effects of nitrogen doses rate on grain yield (GY) and yield components of spring triticale ( $\times$ Tritico secale) cultivars in dry area, based on cultivars plus cultivar $\times$ nitrogen doses interaction GGE biplot and AMMI analysis. The research was designed to evaluate the effects of different nitrogen fertilization levels $\left(0,40,80,120\right.$ and $160 \mathrm{~kg} \mathrm{ha}^{-1}$ of $\left.\mathrm{N}\right)$ on the grain yield and agronomic performance of two cultivars in two growing seasons (2015-16/2016-17). Split plot layout with in randomized complete block design with 3 replications was used in both years. The response to fertilization levels were evaluated through GGE (Genotype main effects and Genotype x Environment interaction) biplot graphic methodologies and regression. Combined analysis of variance of nitrogen applications of two cultivars showed highly significant $(\mathrm{p}<0.01)$ difference between the cultivars, nitrogen applications and interaction. The grain yield of triticale was most of all affected by cultivars (70.8\%) and then by nitrogen doses $(23.5 \%)$ and interaction (5.7\%). On the other hand, the study showed that plant height, number of spikes per $\mathrm{m}^{2}$, number of grain of ears and grain yield increased depending on the increasing nitrogen doses of the cultivars. The highest performance of grain yield of both cultivars were associated with nitrogen $\mathrm{N}_{3}(120 \mathrm{~kg} / \mathrm{ha})$ fertilization in regression and AMMI analysis. The results showed that $80 \mathrm{~kg} / \mathrm{ha} \mathrm{N}$ application can be recommended in triticale traits and grain yield with Esin (new) cultivar. Further more, we found that the GGE biplot method generated highly useful results with high visual quality in the study.
\end{abstract}

Keywords: triticale, nitrogen doses, yield, yield components

\section{Introduction}

Triticale is a new species in field crops and it is expected to be regarded as a seed in the future as well as being evaluated as forage. It is already produced for seed purposes in many African countries. Especially in dry farming areas, where barley and wheat are not well cultivated, in areas where extreme temperatures are experienced and rainfall is insufficient, triticale can be more economically grown than other cereal crops. In these places, triticale is contribute the several advantages in cropping system because cereal grains can use $\mathrm{N}$, captured in soil profile after lentil or chickpea crops. After lentil and chickpea farming it can use $\mathrm{N}$ to accumulate in the soil. In addition, because of the fact that the triticale is abstinent plant, so it is able to get better nitrogen from soil in dry areas compared to wheat and barley. The Syrian border, where the research was carried out, is quite dry and it has become almost impossible to grow wheat without watering. Under these conditions, farmers tend to other cereal crops that may be an alternative to wheat in arid conditions. For this reason, growing triticale is becoming increasingly important (Kendal, 2015; Kendal et al., 2016).

Triticale was designed in order to both biotic and abiotic stressors and thus more suitable for cultivation in marginal areas than wheat (Kendal et al., 2012; Villegas et al., 2010). Triticale is often reported as an interesting product for adverse environmental conditions where environmental factors limit product cultivation (Ugarte et al., 2007; 
Estrada-Campuzano et al., 2008). In such places, each plant does not use the same nitrogen in the same way and the plant cannot make maximum use application amount of nitrogen. Proper N management, are important for obtaining optimum crop yields and positive economic returns while limiting negative environmental impacts of crop production (Gibson et al., 2007). Many authors have reported positive effect of $\mathrm{N}$ fertilization on grain yield and the studies showed that $\mathrm{N}$ fertilization is one of the most important crop management techniques for spring triticale production (Gülmezoglu, Kinaci, 2004; Lestingi et al., 2010). The weather conditions is affect spring triticale grain yield performance and nitrogen $(\mathrm{N})$ fertilization effect total $35.7 \%$ grain yield increase, compared with unfertilized spring triticale (Janušauskaite, 2013).

Nitrogen is needed for early tiller development of plant to set up the crop for a high yield potential. On the other hand; nitrogen fertilization has an important effect on the final harvest, thus if this element is not take from plant, yield is decreases (Moreno et al., 2003). The amount of nitrogen, barley crop needs to reach maximize yield and quality, will depend on the seasonal conditions, soil type, and rotational history of the soil as well as the potential yield of the cultivars. Nitrogen is the key nutrient input for achieving higher yield of triticale (Alazmani, 2015). The farmers have not enough information about use nitrogen fertilizers and adequate information concerning actual soil requirements. Therefore, the study of use $\mathrm{N}$ dozes in triticale cultivars is necessary to recommend optimum nitrogen doses for high yield and yield components in different environment conditions.

The yield of each variety in any environment is a sum of environment $(E)$ main effect, genotype $(\mathrm{G})$ main effect and genotype by environment interaction (GE or GEI) (Farshadfar et al., 2013; Kilic, 2014; Sayar et al., 2013). On the other hand; farmers need varieties that show high performance in terms of yield and other essential agronomic traits by use nitrogen fertilizer. Different statistical analysis, such as correlation, path coefficient and principal component analysis (PCA) can be used to reveal associations between yield and other agronomic traits. The impact of GGE Biplot methods and regression analysis has been clearly showed by different researchers using relationship among factors. This methods; provide the correlative size and interaction (Asfaw et al., 2009; Sayar and Han, 2015; Kendal and Sayar, 2016). So it is very important to identify the use of nitrogen fertilization doses to cultivars for high yield and best quality. The objective of study is to determine the effect of different $\mathrm{N}$ rates on yield, yield components, and to identify optimal $\mathrm{N}$ deses rate using GGE Biplot analysis to recommend doses for application in Southeast Anatolia Region of Turkey.

\section{Material and methods}

The experiment was conducted in the research field of the Kiziltepe Vocational and Training High School, Mardin, Turkey. The experiment was conducted on the basis of split plot layout with completely randomized block design with 3 replications. Main plot was different level of nitrogen fertilizer $\left(0,40,80,120\right.$ and $160 \mathrm{~kg} \mathrm{ha}^{-1}$ of $\mathrm{N}$ and sub plot was different two spring triticale cultivars (Table 1).

This research was conducted in 2015-2016 and 2016-2017 growing seasons. The seeding rates were 500 seeds $\mathrm{m}^{-2}$. Plot size was $7.2 \mathrm{~m}^{2}(1.2 \times 6 \mathrm{~m})$ consisting of 6 rows spaced $20 \mathrm{~cm}$ apart. Sowings were made by using an experimental drill. The fertilization rates for all plots were different $\mathrm{N} \mathrm{ha}^{-1}$ doses and $60 \mathrm{~kg} \mathrm{P}^{-1}$ with sowing time and different $\mathrm{N} \mathrm{ha}^{-1}$ doses was applied to plots in double ridge stage. Harvests were 
made using Hege 140 harvester in $6 \mathrm{~m}^{2}$. Other normal agronomic practices for barley production were followed. During both of growing seasons, heading time (date), spike per square $\left(\mathrm{m}^{2}\right)$, maturity time (date) plant height $(\mathrm{cm})$, length of spike $(\mathrm{cm})$, number of grains per spike, thousand grain weight $(\mathrm{g})$, grain yield $\left(\mathrm{kg} / \mathrm{ha}^{-1}\right)$, hectoliter weight (kg hl), Protein Content (\%) were examined (Kendal and Sayar, 2016). The soil analysis results are shown in Table 2 and the climate data of growing seasons are shown in Table 3 (Mardin Regional Directorate of Meteorology). Different nitrogen use efficiency (NUE, $\left.\mathrm{kg} \mathrm{kg}^{-1} \mathrm{~N}\right)$ was calculated for each treatment: NUEX Trait $=(\mathrm{X}$ trait $\mathrm{N}$ - $\mathrm{X}$ trait $\mathrm{N} 0$ ) / Nx, where $\mathrm{X}$ trait $\mathrm{N}$ is $\mathrm{X}$ trait from nitrogen $(\mathrm{N})$ fertilized treatments, Xtrait N0 - X trait in unfertilized treatment, and Nx- nitrogen input (N40, N80, N120, N160, $\mathrm{kg} \mathrm{ha}^{-1} \mathrm{~N}$ ).

Table 1. Information about cultivars used in the experiment

\begin{tabular}{c|c|c|c}
\hline Cult. name & Where registration & Regist. time & Type \\
\hline Tacettinbey & Cukurova University & 2000 & Spring \\
Esin & GAP International Agricultural and Training Center & 2016 & Spring \\
\hline
\end{tabular}

Table 2. Soil analysis results of trial

\begin{tabular}{c|c|c|c|c|c|c|c}
\hline \multicolumn{3}{c|}{ Texturing (\%) } & \multirow{2}{*}{ pH } & Organic subs. (\%) & $\begin{array}{c}\text { Total P } \\
\text { ppm }\end{array}$ & $\begin{array}{c}\text { Total K } \\
\text { ppm }\end{array}$ & Lime\% \\
\cline { 1 - 3 } Sand & Clay & Mile & & & 22 & 210 & 17.15 \\
\hline 65.6 & 22.4 & 12.0 & 7.5 & 1.4 & 22 & \\
\hline
\end{tabular}

Table 3. Information about meteorological data of location of study

\begin{tabular}{c|c|c|c|c|c|c|c|c|c}
\hline \multirow{2}{*}{ Months } & \multicolumn{3}{|c|}{ Humidity (\%) } & \multicolumn{3}{c|}{ Temperature $\left({ }^{\circ} \mathbf{C}\right)$} & \multicolumn{3}{c}{ Precipitation $(\mathbf{m m})$} \\
\cline { 2 - 10 } & $2015-16$ & $2016-17$ & LTA* & $2015-16$ & $2016-17$ & LTA & $2015-16$ & $2016-17$ & LTA \\
\hline October & 49.6 & 33.3 & 32.9 & 19.5 & 20.5 & 19.3 & 58.2 & 16.5 & 19.7 \\
November & 50.3 & 35.2 & 50.2 & 11.7 & 11.5 & 11.2 & 62.0 & 27.2 & 49.1 \\
December & 51.7 & 71.3 & 47.2 & 6.4 & 3.2 & 4.2 & 64.7 & 128.4 & 58.5 \\
January & 74.1 & 63.6 & 63.7 & 2.2 & 3.1 & 3.2 & 146.3 & 38.3 & 78.7 \\
February & 66.2 & 51.3 & 51.0 & 8.5 & 3.8 & 3.9 & 3.6 & 23.2 & 64.4 \\
March & 59.1 & 62.5 & 62.9 & 10.0 & 9.7 & 9.0 & 119.8 & 101.7 & 99.6 \\
Aprll & 41.3 & 55.7 & 55.2 & 16.8 & 13.5 & 15.2 & 27.1 & 109.2 & 98.5 \\
May & 42.0 & 44.0 & 43.8 & 19.8 & 19.7 & 19.6 & 20.0 & 60.3 & 57.0 \\
June & 28.2 & 26.1 & 25.8 & 26.2 & 26.8 & 26.0 & 1.0 & 0.2 & 2.2 \\
July & 22.4 & 17.0 & 16.5 & 30.6 & 32.4 & 32.1 & 0.1 & 0.0 & 0.6 \\
\hline Total & & & & & & & 502.8 & 505.0 & 528.3 \\
\hline Mean & 48.49 & 46.00 & 44.9 & 15.7 & 14.4 & 14.3 & & & \\
\hline
\end{tabular}

LTA: long term average, *Mardin regional directorate of meteorology station (2017) 
The data obtained from the study related to the investigated grain yield and yield components were analyzed respectively for each year and combined with nitrogen doses by using the JMP 5.0.1 statistical software package (SAS Institute, 2002), and the differences between means were compared using a least significant difference (LSD) test at the 0.05 probability level (Steel and Torrie, 1980). Also regression analysis was done by this program. On the other hand; GGE biplot analyses were carried out using GGE biplot software to show the differences among the applied nitrogen doses and crop characteristics and cultivar crop characteristics in two growing seasons (Dogan et al., 2016; Kilic, 2016). GGE biplot analysis also allows comparison amongst nitrogen doses in terms of their discriminating ability and representativeness. These values can be assessed using the discriminating power of the doses' biplot screen of the GGE biplot (Yan and Thinker, 2006). With the AMMI biplot analysis graph in the study: It was aimed at illustrating grain yield performance of $\mathrm{N}$ doses and status of triticale cultivars (Fig. 1b), grain yield performance of cultivar (Fig. 1c), the effect of $\mathrm{N}$ doses on grain yield on years (Fig. 1d). Also, with the GGE biplot analysis graphs in the study: It was aimed at revealing relation among examined doses and traits (Fig. 1e), sector analysis of doses and grouping traits (Fig. If), ranking of doses on traits (Fig. 1g), comparison of doses on traits (Fig. 1h)

\section{Results}

The combined ANOVA revealed highly significant differences among the years, cultivars and interaction of them for all components $(\mathrm{P}<0.01,0.05)$, the differences among nitrogen doses were highly significant $(\mathrm{P}<0.01,0.05)$ for all components as shown in Tables 4 and 5. Generally, breeders interested in the genotypes with high genotypic main effect (average over years and nitrogen doses) and with low fluctuation in yield or yield components (stable). On the other hand, the study showed that plant height, number of spikes per $\mathrm{m}^{2}$, number of grain of ears and grain yield increased depending on the increasing nitrogen doses of the cultivars.

\section{The results of the data reviewed}

Table 4. Variance of analysis on grain yield and yield components of triticale

\begin{tabular}{c|c|c|c|c|c|c|c|c|c|c|c}
\hline Sources & $\mathbf{D F}$ & $\begin{array}{c}\mathbf{H T} \\
(\mathbf{d a t e})\end{array}$ & $\begin{array}{c}\mathbf{S S} \\
\left(\mathbf{m}^{2}\right)\end{array}$ & $\begin{array}{c}\mathbf{M T} \\
(\mathbf{d a t e})\end{array}$ & $\begin{array}{c}\mathbf{P H} \\
(\mathbf{c m})\end{array}$ & $\begin{array}{c}\mathbf{S L} \\
(\mathbf{c m})\end{array}$ & $\mathbf{N G S}$ & $\begin{array}{c}\mathbf{T G W} \\
(\mathbf{g})\end{array}$ & $\begin{array}{c}\mathbf{G Y} \\
\left(\mathbf{k g} / \mathbf{h a}^{-1}\right)\end{array}$ & $\begin{array}{c}\mathbf{H L} \\
(\mathbf{k g} / \mathbf{h l} \mathbf{1})\end{array}$ & $\begin{array}{c}\mathbf{P C} \\
(\mathbf{\%})\end{array}$ \\
\hline Year & 1 & $400.4^{* *}$ & $1696.0^{*}$ & $589.1^{* *}$ & $779.0^{*}$ & $3.3 \mathrm{~ns}$ & $477.7^{*}$ & $0.9 \mathrm{~ns}$ & $522.2 \mathrm{~ns}$ & $123.8^{*}$ & $0.3 \mathrm{~ns}$ \\
Error 1 & 4 & 0.7 & 413.9 & 0.9 & 36.7 & 3.5 & 14.4 & 9.1 & 4322.9 & 4.4 & 3.6 \\
Cultivar & 1 & $88.8^{* *}$ & $380.0 \mathrm{~ns}$ & $26.7^{* *}$ & $449.4^{* *}$ & $17.1^{* *}$ & $492.5^{* *}$ & $212.8^{* *}$ & $158620.0^{* *}$ & $13.1^{*}$ & $1.5^{*}$ \\
Year*Cult. & 1 & $4.8^{* *}$ & $498.8 \mathrm{~ns}$ & $6.7^{* *}$ & $2.5 \mathrm{~ns}$ & $2.4 \mathrm{~ns}$ & $273.5^{* *}$ & $28.8^{* *}$ & $84.0 \mathrm{~ns}$ & $86.9^{* *}$ & $1.3^{*}$ \\
Nitr. Doses & 4 & $2.4^{*}$ & $7083.2^{* *}$ & $2.5^{*}$ & $2607.4^{* *}$ & $58.9^{* *}$ & $391.5^{* *}$ & $20.4^{*}$ & $52602.6^{* *}$ & $33.4^{*}$ & $5.9^{*}$ \\
Year*N.Dos & 4 & $1.2 \mathrm{~ns}$ & $5371.2^{*}$ & $1.1^{*}$ & $132.1^{*}$ & $11.6^{*}$ & $69.5^{*}$ & $3.2 \mathrm{~ns}$ & $8718.4^{* *}$ & $18.2^{*}$ & $0.5 \mathrm{~ns}$ \\
Cul.*N.Dos & 4 & $0.4 \mathrm{~ns}$ & $130.9 \mathrm{~ns}$ & $0.5 \mathrm{~ns}$ & $57.9 \mathrm{~ns}$ & $10.8 \mathrm{~ns}$ & $47.3 \mathrm{~ns}$ & $40.3^{* *}$ & $12757.2^{* *}$ & $12.5^{*}$ & $1.1 \mathrm{~ns}$ \\
Y*CND & 4 & $0.1 \mathrm{~ns}$ & $1247.4 \mathrm{~ns}$ & $0.8 \mathrm{~ns}$ & $120.6^{*}$ & $2.8 \mathrm{~ns}$ & $18.7 \mathrm{~ns}$ & $10.8 \mathrm{~ns}$ & $410.9 \mathrm{~ns}$ & $19.5^{*}$ & $1.2 \mathrm{~ns}$ \\
Error 2 & 36 & 5.9 & 6039.5 & 3.7 & 267.8 & 23.1 & 143.8 & 49.6 & 6022.4 & 40.5 & 7.5 \\
C. Total & 59 & 504.9 & 22861.0 & 632.0 & 4453.4 & 133.4 & 1928.9 & 376.0 & 244061.0 & 352.2 & 23.0 \\
CV(\%) & & 0.24 & 9.18 & 1.03 & 2.52 & 7.22 & 4.37 & 3.14 & 3.36 & 1.37 & 4.22 \\
\hline
\end{tabular}

HT: Heading Time, SS: Spike ofper Square, MT: Maturity time, PH: Plant Height, LS: Length of Spike, NGS: Number of Grains per Spike, TGW: Thousand Grain Weight, GY: Grain Yield, HL: Hectoliter weight, PC: Protein Content 
Table 5. The variance of AMMI analysis on grain yield of nitrogen doses

\begin{tabular}{c|c|c|c|c|c}
\hline Source & Df & SS & MS & F & Explained SS (\%) \\
\hline Total & 59 & 244061 & 4137 & & \\
Treatments & 9 & 223980 & 24887 & 70.23 & \\
Cultivar & 1 & 158620 & 158620 & $447.65^{* *}$ & 70.8 \\
N doses & 4 & 52603 & 13151 & $22.26^{* *}$ & 23.5 \\
Block & 10 & 5907 & 591 & 1.67 & \\
Interactions & 4 & 12757 & 3189 & $9.00^{* *}$ & 5.7 \\
IPCA1 & 4 & 12757 & 3189 & $9.00^{* *}$ & \\
Error & 40 & 14174 & 354 & & \\
\hline
\end{tabular}

Df: degrees of freedom; **: $\mathrm{p}<0.01 ; *: \mathrm{P}<0.05$

The mean grain yield (GY) of 2015/16 growing season $\left(3878 \mathrm{~kg} \mathrm{ha}^{-1}\right)$ was higher than that of $2016 / 17\left(3819 \mathrm{~kg} \mathrm{ha}^{-1}\right)$, the mean yield of growing seasons was changed from $3334 \mathrm{~kg} \mathrm{ha}^{-1}$ (Esin) to $4362 \mathrm{~kg} \mathrm{ha}^{-1}$ (Tacettinbey) in Table 6.

Table 6. The data effect interaction of nitrogen levels and triticale cultivars

\begin{tabular}{|c|c|c|c|c|c|c|c|c|c|c|c|c|c|c|}
\hline \multirow{2}{*}{ Cultivar } & & \multicolumn{5}{|c|}{ N Doses } & \multirow{2}{*}{ Mean } & & \multicolumn{5}{|c|}{ N Doses } & \multirow{2}{*}{ Mean } \\
\hline & & $\mathbf{N}_{0}$ & $\mathrm{~N}_{1}$ & $\mathbf{N}_{2}$ & $\mathbf{N}_{3}$ & $\mathbf{N}_{4}$ & & & $\mathbf{N}_{0}$ & $N_{1}$ & $\mathbf{N}_{2}$ & $\mathbf{N}_{3}$ & $\mathbf{N}_{4}$ & \\
\hline Tacettinbey & Ð & 163.5 & 163.8 & 163.8 & 163.8 & 164.2 & & & $39.0^{\mathrm{d}}$ & $40.9^{\mathrm{cd}}$ & $42.6^{\mathrm{c}}$ & $46.0^{\mathrm{b}}$ & $46.0^{\mathrm{b}}$ & 42. \\
\hline Esin & $\ddot{\sigma}$ & 166.0 & 166.0 & 166.5 & 166.3 & 166.5 & $166.3^{\mathrm{A}}$ & 0 & $43.2^{\mathrm{c}}$ & $49.5^{\mathrm{a}}$ & $50.2^{\mathrm{a}}$ & $51.0^{\mathrm{a}}$ & $48.8^{\mathrm{a}}$ & $48.6^{\mathrm{A}}$ \\
\hline Mean & $\underline{\Xi}$ & $164.8^{\mathrm{C}}$ & $164.9^{\mathrm{BC}}$ & $165.2^{\mathrm{AB}}$ & $165.1^{\mathrm{AC}}$ & $165.3^{\mathrm{A}}$ & & & $41.1 \mathrm{D}$ & $45.2^{\mathrm{C}}$ & $46.4^{\mathrm{BC}}$ & $48.5^{\mathrm{A}}$ & $47.4^{\mathrm{AB}}$ & \\
\hline Tacettinbey & I & 125.8 & 134.0 & 140.2 & 137.7 & 154.8 & 138.5 & & $34.9^{\text {cd }}$ & $35.9^{\mathrm{cd}}$ & $36.0^{\mathrm{c}}$ & $35.8^{\mathrm{cd}}$ & $34.5^{\mathrm{d}}$ & \\
\hline & 刍 & 128.7 & 136.7 & 144.0 & 142.8 & 165.5 & 143.5 & 5 & $36.4^{\mathrm{b}}$ & $37.2^{\mathrm{b}}$ & $37.1^{\mathrm{b}}$ & $38.0^{\mathrm{a}}$ & $38.2^{\mathrm{a}}$ & $39.2^{\mathrm{A}}$ \\
\hline Mean & 2 & $127.2^{\mathrm{C}}$ & $135.3^{\mathrm{BC}}$ & $142.1^{\mathrm{B}}$ & $140.3^{\mathrm{B}}$ & $160.2^{\mathrm{A}}$ & & & $36.4^{\mathrm{C}}$ & $36.9^{\mathrm{BC}}$ & $37.3^{\mathrm{A}} \mathrm{C}$ & $38.0^{\mathrm{A}}$ & $37.8^{\mathrm{AB}}$ & \\
\hline Гacettinbey & Ð & 30.0 & 30.2 & 30.3 & 30.5 & 30.7 & $31.7^{\mathrm{A}}$ & ב & $2948^{h}$ & $3542^{\mathrm{ef}}$ & $3408^{\mathrm{fg}}$ & $3485^{f}$ & $3287^{\mathrm{g}}$ & \\
\hline $\mathrm{E}$ & हु & 31.5 & 31.5 & 31.8 & 31.5 & 32.0 & $30.3^{\mathrm{B}}$ & $\underset{2}{20}$ & $364.8^{\mathrm{e}}$ & $4200^{\mathrm{d}}$ & $4657^{\mathrm{b}}$ & $4847^{\mathrm{a}}$ & $4460^{c}$ & $4362^{\mathrm{A}}$ \\
\hline Mean & $\Sigma$ & $30.8^{\mathrm{C}}$ & $30.8^{\mathrm{BC}}$ & $31.1^{\mathrm{AB}}$ & $31.0^{\mathrm{BC}}$ & $31.3^{\mathrm{A}}$ & & U & $329.8^{\mathrm{D}}$ & $3871^{C}$ & $4033^{\mathrm{B}}$ & $4166^{\mathrm{A}}$ & $3873^{\mathrm{C}}$ & \\
\hline Tacettin & $\widehat{\approx}$ & 95.9 & 100.5 & 103.5 & 112.5 & 114.7 & $105.4^{\mathrm{B}}$ & & $74.3^{b}$ & $76.9^{\mathrm{a}}$ & $76.9^{\mathrm{a}}$ & $77.2^{\mathrm{a}}$ & $77.6^{\mathrm{a}}$ & $76.6^{\mathrm{B}}$ \\
\hline Esin & है & 102.1 & 105.6 & 112.0 & 117.5 & 117.2 & $110.9^{\mathrm{A}}$ & 3 & $77.0^{\mathrm{a}}$ & $77.6^{\mathrm{a}}$ & $77.4^{\mathrm{a}}$ & $78.0^{\mathrm{a}}$ & $77.7^{\mathrm{a}}$ & $77.5^{\mathrm{A}}$ \\
\hline Mean & $\underline{a}$ & $99.0^{\mathrm{D}}$ & $103.1^{\mathrm{C}}$ & $107.7^{\mathrm{B}}$ & $115.0^{\mathrm{A}}$ & $116.0^{\mathrm{A}}$ & & & $75 .^{6 \mathrm{~B}}$ & $77.3^{\mathrm{A}}$ & $77.2^{\mathrm{A}}$ & $77.6^{\mathrm{A}}$ & $77.6^{\mathrm{A}}$ & \\
\hline Tacettinh & $=$ & $8.3^{\mathrm{f}}$ & $10.2^{\mathrm{e}}$ & $10.7^{\mathrm{de}}$ & $12.3^{\mathrm{ab}}$ & $11.3^{\text {cd }}$ & $10.6^{\mathrm{B}}$ & 2 & 10.5 & 11.1 & 10.8 & 11.5 & 11.1 & $11.0^{\mathrm{A}}$ \\
\hline & & $10.3^{\mathrm{e}}$ & $11.5^{\mathrm{bd}}$ & $12.5^{\mathrm{a}}$ & $12.2^{\mathrm{bc}}$ & $11.7^{\mathrm{bc}}$ & $11.6^{\mathrm{A}}$ & 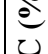 & 10.2 & 10.3 & 10.8 & 11.0 & 11.0 & $10.7^{\mathrm{B}}$ \\
\hline Mean & & $9.3^{\mathrm{D}}$ & $10.8^{\mathrm{C}}$ & $11.6^{\mathrm{B}}$ & $12.3^{\mathrm{A}}$ & $11.5^{\mathrm{B}}$ & & $a$ & $10.3^{\mathrm{C}}$ & $10.7^{\mathrm{BC}}$ & $10.8^{\mathrm{B}}$ & $11.3^{\mathrm{A}}$ & $11.0^{\mathrm{AB}}$ & \\
\hline
\end{tabular}

HT: Heading Time, SS: Spike of per Square, MT: Maturity time, PH: Plant Height, LS: Length of Spike,

$\mathrm{N}$ Doses $-\mathrm{N}_{0}$ : not applied, $\mathrm{N}_{1}: 40 \mathrm{~kg} / \mathrm{ha}, \mathrm{N}_{2}: 80 \mathrm{~kg} / \mathrm{ha}, \mathrm{N}_{3}: 120 \mathrm{~kg} / \mathrm{ha} \mathrm{N}_{4}: 160 \mathrm{~kg} / \mathrm{ha}$

The yield of application nitrogen doses were ranged from $3298 \mathrm{~kg} \mathrm{ha}^{-1}\left(\mathrm{~N}_{0}\right)$ to $4166 \mathrm{~kg} \mathrm{ha}^{-1}\left(\mathrm{~N}_{3}\right)$. The yield of cultivar and nitrogen interaction were changed from $2948 \mathrm{~kg} \mathrm{ha}^{-1}$ to $4847 \mathrm{~kg} \mathrm{ha}^{-1}$ and the best yield was obtained by $\mathrm{N}_{3}$ nitrogen doses in Esin, while the low yield obtained from $\mathrm{N}_{0}$ (without nitrogen) doses and Tacettinbey variety (Table 7). 
Table 7. Influence of different nitrogen levels on yield and yield components of triticale cultivars

\begin{tabular}{|c|c|c|c|c|c|c|c|c|c|c|c|c|c|}
\hline \multirow{2}{*}{ Cultivar } & & \multicolumn{5}{|c|}{ 2015-2016 } & \multirow[t]{2}{*}{ Mean } & \multicolumn{5}{|c|}{ 2016-2017 } & \multirow[t]{2}{*}{ Mean } \\
\hline & & No & N1 & N2 & N3 & N4 & & No & N1 & N2 & N3 & N4 & \\
\hline Tacettinbey & \multirow{4}{*}{ 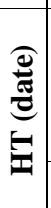 } & 161.0 & 161.3 & 161.7 & 161.7 & 162.0 & $161.5^{\mathrm{D}}$ & 166 & 166.3 & 166 & 166 & 166.3 & $166.1^{\mathrm{B}}$ \\
\hline Esin & & 163.0 & 163.0 & 163.7 & 163.7 & 163.7 & $163.4^{\mathrm{C}}$ & 169 & 169 & 169.3 & 169 & 169.3 & $169.1^{\mathrm{A}}$ \\
\hline $\mathrm{N}$ mean & & $162.0^{\mathrm{c}}$ & $162.2^{\mathrm{c}}$ & $162.7^{\mathrm{b}}$ & $162.7^{\mathrm{b}}$ & $162.8^{\mathrm{b}}$ & & $167.5^{\mathrm{a}}$ & $167.7^{\mathrm{a}}$ & $167.7^{\mathrm{a}}$ & $167.5^{\mathrm{a}}$ & $167.8^{\mathrm{a}}$ & \\
\hline Year mean & & \multicolumn{5}{|c|}{$162.5^{\mathrm{B}}$} & & \multicolumn{5}{|c|}{$167.6^{\mathrm{A}}$} & \\
\hline Tacettinbey & \multirow{4}{*}{$\begin{array}{l}\text { 五 } \\
\tilde{\sigma} \\
\tilde{s}\end{array}$} & 121.0 & 124.7 & 134.0 & 139.3 & 161.3 & 136.1 & 130.7 & 143.3 & 146.3 & 136 & 148.3 & 140.9 \\
\hline Esin & & 107.0 & 110.3 & 138.7 & 147.0 & 173.7 & 135.3 & 150.3 & 163 & 149.3 & 138.7 & 157.3 & 151.7 \\
\hline $\mathrm{N}$ mean & & $114.0^{\mathrm{d}}$ & $117.5^{\mathrm{d}}$ & $136.3^{\mathrm{c}}$ & $143.2^{\mathrm{bc}}$ & $167.5^{\mathrm{a}}$ & & $140.5^{\mathrm{bc}}$ & $153.2^{\mathrm{ab}}$ & $147.8^{\mathrm{bc}}$ & $137.3^{\mathrm{c}}$ & $152.8^{\mathrm{ab}}$ & \\
\hline Year mean & & \multicolumn{5}{|c|}{$135.7^{\mathrm{B}}$} & & \multicolumn{5}{|c|}{$146.3^{\mathrm{A}}$} & \\
\hline Tacettinbey & \multirow{4}{*}{ 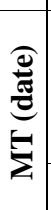 } & 27.0 & 27.0 & 27.7 & 28.0 & 28.0 & $27.5^{\mathrm{D}}$ & 33.0 & 33.3 & 33.0 & 33.0 & 33.33 & $33.1^{\mathrm{B}}$ \\
\hline Esin & & 28.0 & 28.0 & 28.3 & 28.0 & 28.7 & $28.2^{\mathrm{C}}$ & 35.0 & 35.0 & 35.3 & 35.0 & 35.33 & $35.1^{\mathrm{A}}$ \\
\hline $\mathrm{N}$ mean & & $27.5^{\mathrm{c}}$ & $27.5^{\mathrm{c}}$ & $28.0^{\mathrm{b}}$ & $28.0^{\mathrm{b}}$ & $28.3^{\mathrm{b}}$ & & $34.0^{\mathrm{a}}$ & $34.2^{\mathrm{a}}$ & $34.2^{\mathrm{a}}$ & $34.0^{\mathrm{a}}$ & $34.3^{\mathrm{a}}$ & \\
\hline Year mean & & \multicolumn{5}{|c|}{$27.9^{\mathrm{B}}$} & & \multicolumn{5}{|c|}{$34.1^{\mathrm{A}}$} & \\
\hline Tacettinbey & \multirow{4}{*}{ 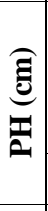 } & $92.1^{1}$ & $95.3^{\text {h1 }}$ & $96.9^{\mathrm{h}}$ & $111.6^{\mathrm{df}}$ & $112.1^{\mathrm{de}}$ & 101.6 & $99.7^{\mathrm{h}}$ & $105.7^{\mathrm{g}}$ & $110.0^{\mathrm{eg}}$ & $113.3^{\mathrm{ce}}$ & $117.3^{\text {bd }}$ & 109.2 \\
\hline Esin & & $96.9^{\mathrm{h}}$ & $99.3^{\mathrm{h}}$ & $110.9^{\mathrm{ef}}$ & $114.3^{\mathrm{be}}$ & $116.0^{\text {bd }}$ & 107.5 & $107.3^{\mathrm{fg}}$ & $112.0^{\mathrm{de}}$ & $113.0^{\mathrm{ce}}$ & $120.7^{\mathrm{a}}$ & $118 .^{3 \mathrm{ab}}$ & 114.27 \\
\hline $\mathrm{N}$ mean & & $94.5^{\mathrm{F}}$ & $97.3^{\mathrm{F}}$ & $103.9^{\mathrm{E}}$ & $112.9^{\mathrm{C}}$ & $114.1^{\mathrm{BC}}$ & & $103.5^{\mathrm{E}}$ & $108.8^{\mathrm{D}}$ & $111.5^{\mathrm{CD}}$ & 117. ${ }^{\mathrm{AB}}$ & $117.8^{\mathrm{A}}$ & \\
\hline Year mean & & \multicolumn{5}{|c|}{$104.5^{\mathrm{B}}$} & & \multicolumn{5}{|c|}{$111.7^{\mathrm{A}}$} & \\
\hline Tacettinbey & \multirow{4}{*}{$\begin{array}{c}\underset{\mathrm{E}}{\tilde{E}} \\
\vec{a}\end{array}$} & 8.0 & 9.3 & 9.7 & 12.3 & 11.3 & 10.1 & 8.7 & 11.0 & 11.7 & 12.3 & 11.3 & 11.0 \\
\hline Esin & & 9.7 & 11.0 & 12.3 & 13.0 & 12.0 & 11.6 & 11.0 & 12.0 & 12.7 & 11.3 & 11.3 & 11.7 \\
\hline $\mathrm{N}$ mean & & $8.8^{\mathrm{F}}$ & $10.2^{\mathrm{DE}}$ & $11.0^{\mathrm{CD}}$ & $12.7^{\mathrm{A}}$ & $11.7^{\mathrm{BC}}$ & & $9.8^{\mathrm{E}}$ & $11.5^{\mathrm{BC}}$ & $12.2^{\mathrm{AB}}$ & $11.8^{\mathrm{AC}}$ & $11.3^{\mathrm{BC}}$ & \\
\hline Year mean & & & & 10.9 & & & & & & 11.3 & & & \\
\hline Tacettinbey & \multirow{4}{*}{$\mathscr{Z}$} & 38.6 & 41.3 & 41.5 & 44.6 & 44.8 & $42.2^{\mathrm{B}}$ & 40.3 & 44.7 & 45.0 & 44.0 & 43.7 & $43.5^{\mathrm{B}}$ \\
\hline Esin & & 39.4 & 40.5 & 43.7 & 47.3 & 47.2 & $43.6^{\mathrm{B}}$ & 46.0 & 54.3 & 55.3 & 58.0 & 54.0 & $53.5^{\mathrm{A}}$ \\
\hline $\mathrm{N}$ mean & & $39.5^{\mathrm{e}}$ & $43.0^{\mathrm{d}}$ & $43.2^{\mathrm{d}}$ & $44.3^{\mathrm{d}}$ & $44.3^{\mathrm{d}}$ & & $42.7^{\mathrm{d}}$ & $47.4^{\mathrm{c}}$ & $49.5^{\mathrm{bc}}$ & $52.7^{\mathrm{a}}$ & $50.6^{\mathrm{ab}}$ & \\
\hline Year mean & & \multicolumn{5}{|c|}{$43.0 \mathrm{~B}$} & & \multicolumn{5}{|c|}{$48.5^{\mathrm{A}}$} & \\
\hline Tacettinbey & & 36.1 & 36.6 & 36.9 & 35.9 & 34.3 & $36.0^{\mathrm{C}}$ & 33.6 & 35.1 & 35.1 & 35.6 & 34.7 & $34.8^{\mathrm{D}}$ \\
\hline Esin & 兽 & 36.6 & 36.5 & 38.3 & 39.8 & 40.5 & $38.3^{\mathrm{B}}$ & 39.2 & 39.3 & 39.1 & 40.5 & 41.8 & $40.0^{\mathrm{A}}$ \\
\hline $\mathrm{N}$ mean & 3 & 36.4 & 36.6 & 37.6 & 37.9 & 37.4 & & 36.4 & 37.2 & 37.1 & 38.0 & 38.2 & \\
\hline Year mean & 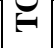 & & & 37.2 & & & & & & 37.4 & & & \\
\hline Tacettinbey & & 3087 & 3773 & 3403 & 3410 & 3203 & $3375^{\mathrm{B}}$ & 2810 & 3310 & 3413 & 3560 & 3370 & $3293^{\mathrm{B}}$ \\
\hline Esin & $\frac{\pi}{90}$ & 3667 & 4437 & 4687 & 4760 & 4350 & $4380^{\mathrm{A}}$ & 3630 & 3963 & 4627 & 4933 & 4570 & $4345^{\mathrm{A}}$ \\
\hline $\mathrm{N}$ mean & $\begin{array}{l}\stackrel{y}{z} \\
\nu\end{array}$ & $3377^{\mathrm{D}}$ & $4105^{\mathrm{AB}}$ & $4045^{\mathrm{B}}$ & $4085^{\mathrm{B}}$ & $3777^{\mathrm{C}}$ & & $3220^{\mathrm{E}}$ & $3637^{\mathrm{C}}$ & $4020^{\mathrm{B}}$ & $4247^{\mathrm{A}}$ & $3970^{\mathrm{B}}$ & \\
\hline Year mean & & & & 3878 & & & & & & 3819 & & & \\
\hline Tacettinbey & 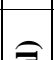 & $75.4^{\mathrm{df}}$ & $76.7^{\mathrm{ce}}$ & $76.8 \mathrm{ce}$ & $75.2^{\text {ef }}$ & $77.6^{\text {be }}$ & $76.3^{B}$ & $73.1^{\mathrm{g}}$ & $77.1^{\mathrm{cd}}$ & $77.1^{\mathrm{cd}}$ & $79.3^{\mathrm{cd}}$ & $77.5^{\mathrm{ab}}$ & $76.8^{\mathrm{B}}$ \\
\hline Esin & $\begin{array}{l}7 \\
800 \\
z 0\end{array}$ & $74.3^{\mathrm{fg}}$ & $74.5^{\mathrm{fg}}$ & $74.3 f g$ & $75.5^{\mathrm{df}}$ & $75.7^{\mathrm{df}}$ & $74.9^{\mathrm{C}}$ & $79.6^{\mathrm{a}}$ & $80.7^{\mathrm{a}}$ & $80.4^{\mathrm{a}}$ & $80.5^{\mathrm{a}}$ & $79.6^{\mathrm{a}}$ & $80.2^{\mathrm{A}}$ \\
\hline $\mathrm{N}$ mean & 3 & $74.9^{\mathrm{E}}$ & $75.6^{\mathrm{CE}}$ & $75.6^{\mathrm{CE}}$ & $75.3^{\mathrm{DE}}$ & $76.7^{\mathrm{C}}$ & & $76.3^{\mathrm{CD}}$ & $78.9^{\mathrm{AB}}$ & $78.8^{\mathrm{AB}}$ & $79.9^{\mathrm{A}}$ & $78.6^{\mathrm{B}}$ & \\
\hline Year mean & $\pi$ & & & $75.6^{\mathrm{B}}$ & & & & & & $78.5^{\mathrm{A}}$ & & & \\
\hline Tacettinbey & & 10.2 & 10.8 & 10.6 & 11.4 & 10.9 & $10.8^{\mathrm{B}}$ & 10.7 & 11.3 & 11.0 & 11.6 & 11.4 & $11.2^{\mathrm{A}}$ \\
\hline Esin & 8 & 10.6 & 10.5 & 10.6 & 10.9 & 11.1 & $10.7^{\mathrm{B}}$ & 9.8 & 10.1 & 11.1 & 11.1 & 10.8 & $10.6^{\mathrm{B}}$ \\
\hline $\mathrm{N}$ mean & U & 10.4 & 10.7 & 10.6 & 11.1 & 11.0 & & 10.3 & 10.7 & 11.0 & 11.4 & 11.1 & \\
\hline Year mean & & & & 10.8 & & & & & & 10.9 & & & \\
\hline
\end{tabular}

NGS: Number of Grains per Spike, TGW: Thousand Grain Weight, GY: Grain Yield, HL: Hectoliter Weight, PC:

Protein Content. N Doses $-\mathrm{N}_{0}$ : not applied, $\mathrm{N}_{1}: 40 \mathrm{~kg} / \mathrm{ha}, \mathrm{N}_{2}: 80 \mathrm{~kg} / \mathrm{ha}, \mathrm{N}_{3}: 120 \mathrm{~kg} / \mathrm{ha}, \mathrm{N}_{4}: 160 \mathrm{~kg} / \mathrm{ha}$ 
The results of grain yield showed that first growing season had high yield than the second year and Esin variety was yielding than Tacettinbey during two grooving seasons. The results of grain yield showed that first growing season had higher yield than the second year and Esin variety changed from $4760 \mathrm{~kg} / \mathrm{hl}$ to $4933 \mathrm{~kg} / \mathrm{hl}\left(\mathrm{N}_{3^{-}}\right.$ 2015/16-2016/17) had a higher yield Tacettinbey (2810-3087 kg/hl, $\mathrm{N}_{0}$ ) during two growing seasons in Table 7. Nitrogen use efficiency (NUE) was considerably influenced by cultivars, using $\mathrm{N}$ doses and growing seasons. The main factor determining the differences in NUE yield of cultivars was changed from $13.1 \%$ (Tacettinbey) to $19.6 \%$ (Esin) (Table 7). The influence of growing season on NUE yield was changed from 21.6(2015-16) to $23.3 \%$ (2016-17). The highest $\mathrm{NUE}_{\text {yield }}$ of interaction was obtained from $\mathrm{N}_{3}$ fertilization rate (35.9\%) in 2016-17 growing season. As expected, depend on increasing basic $\mathrm{N}$ fertilization rate increased $\mathrm{NUE}_{\text {yield. }}$ Each $\mathrm{N}$ rate increasing by $40 \mathrm{~kg}$ increased NUE $\mathrm{N}_{\text {yield }}$ by $2.5 \mathrm{~kg} \mathrm{~kg}^{-1}$.

The mean thousand grain weight (TGW) of 2016/17 growing season $(37.4 \mathrm{~g})$ was high than 2015/16 (37.2 g), the mean thousand grain weight of cultivars of both years was changed from $34.8 \mathrm{~g}$ (Tacettinbey) to $40.0 \mathrm{~g}$ (Esin) in Table 6. The number of thousand grain weight of nitrogen doses and cultivar interaction of both year means were changed from $36.4 \mathrm{~g}\left(\mathrm{~N}_{0}\right)$ to $38.2 \mathrm{~g}\left(\mathrm{~N}_{4-}\right.$ 2016/17). The application nitrogen doses of thousand grain weight were ranged from $36.4 \mathrm{~g}\left(\mathrm{~N}_{0}\right)$ to $38.0 \mathrm{~g}\left(\mathrm{~N}_{3}\right)$ in Table 7. The thousand grain weight was increased depend on nitrogen rate till $\mathrm{N}_{3}$ application doses for both varieties.

The mean hectoliter weight $(\mathrm{HW})$ of $2016 / 17$ growing season $(78.5 \mathrm{~kg} / \mathrm{hl})$ was high than $2015 / 16(75.6 \mathrm{~kg} / \mathrm{hl})$, the mean hectoliter weight of cultivars of both years was changed from $76.8 \mathrm{~kg} / \mathrm{hl}$ (Tacettinbey) to $80.2 \mathrm{~kg} / \mathrm{hl}$ (Esin) in Table 6. The number of hectoliter weight of nitrogen doses and cultivar interaction of both year means were changed from $74.9 \mathrm{~kg} / \mathrm{hl}\left(\mathrm{N}_{0}-2015 / 16\right)$ to $79.9 \mathrm{~g}\left(\mathrm{~N}_{3}-2016 / 17\right)$. The application nitrogen doses of hectoliter weight were ranged from $75.6 \mathrm{~kg} / \mathrm{hl}\left(\mathrm{N}_{0}\right)$ to $77.6 \mathrm{~kg} / \mathrm{hl}\left(\mathrm{N}_{3}\right.$ and $\left.\mathrm{N}_{4}\right)$ in Table 7. The hectoliter weight was increased depend on nitrogen rate till $\mathrm{N}_{4}$ doses for both varieties. N3 and $\mathrm{N} 4$ doses had the same values, so the $\mathrm{N}_{3}$ dose application is better for hectoliter weight in tiriticale.

The mean protein content (PC) of 2016/17 growing season (10.9\%) was higher than $2015 / 16(10.8 \%)$, the mean hectoliter weight of cultivars of both years was changed from $11.2 \%$ (Tacettinbey) to $10.6 \%$ (Esin) in Table 6 . The number of protein content of nitrogen doses and cultivar interaction of both year means were changed from $10.3 \%$ $\left(\mathrm{N}_{0}-2016 / 17\right)$ to $11.4 \%\left(\mathrm{~N}_{3}-2016 / 17\right)$. The application nitrogen doses of protein content were ranged from $10.3 \%\left(\mathrm{~N}_{0}\right)$ to $11.3 \%\left(\mathrm{~N}_{3}\right)$ in Table 7 . The protein content was increased depend on nitrogen rate till $\mathrm{N}_{3}$ doses for both varieties. Nitrogen use efficiency (NUE) of protein content influenced by cultivars, using $\mathrm{N}$ doses and growing seasons. The main factor determining the differences in $\mathrm{NUE}_{\text {protein }}$ of cultivars was changed from $5.3 \%$ (Tacettinbey) to $4.2 \%$ (Esin) (Table 7). The influence of growing season on $\mathrm{NUE}_{\text {protein }}$ was changed from $4 \%$ (2015-16) to 7\% (2016-17). The highest $\mathrm{NUE}_{\text {protein }}$ of interaction was obtained from N3 fertilization rate (10.7\%) in 2016-17 growing season. As expected, depend on increasing basic $\mathrm{N}$ fertilization rate increased $\mathrm{NUE}_{\text {protein. }}$ Each $\mathrm{N}$ rate increasing by $40 \mathrm{~kg}$ increased $\mathrm{NUE}_{\text {protein }}$ by $1.5-2.5 \mathrm{~kg} \mathrm{~kg}^{-1}$ till N3 fertilization rate

The heading time (HT) was calculated from 01 January to heading time as day for both varieties separately in Table 7. The maturity time (MT) was calculated from heading time to maturity time as day for both varieties separately. On the other hand, 
ear per square (SS), plant height (PH), long of spike (LE), number of grain per spike (NGS) were recorded and results were showed in Table 7. The results showed that there is positive correlation between heading time and maturity time, and depend on $\mathrm{N}$ application doses heading time and maturity time are delayed. The cultivars are affected the same on application doses. On the other hand the study indicated the ear per square (SS) and grain per spike (NGS) are contributing the grain yield. Moreover, all traits results (HT, MT, LS, NGS and SS) were increased depend on nitrogen rate till N3 doses for varieties, years and interaction.

\section{AMMI and GGE biplot analysis}

Analysis of variance for nitrogen doses (ND) component (C), and the cultivar (C) $\times$ component $(\mathrm{C})$ interaction showed significant $(\mathrm{P}<0.01, \mathrm{P}<0.01)$ effect, and the total sum of squares explained for $96.15 \%$, with PC1 and PC2 accounting $85.03 \%$ and $11.12 \%$ for nitrogen doses (ND) component (C) (Fig. 1e-h), Moreover, the AMMI analysis showed that the grain yield of triticale was most of all affected by cultivars (70.8\%) and then by nitrogen doses (23.5\%) and interaction $(5.7 \%)$.

AMMI Analysis was done on grain yield for interaction nitrogen doses, cultivars and years (Fig. 1b-e). The results of interaction nitrogen doses and cultivars of grain yield (Fig. 1b) showed that grain yield is increased depend on nitrogen doses (Fig. 1a) till $\mathrm{N}_{3}$ application doses, the contribute of $\mathrm{N}_{1}$ and $\mathrm{N}_{4}$ to grain yield of triticale is the same, but $\mathrm{N}_{3}$ application dose is contribute to grain yield in highest level. The interaction of $\mathrm{N}$ doses and years showed that the highest grain yield was obtained from the first year (Fig. 1c), and the effect of $\mathrm{N}_{3}$ doses on grain yield was highest than other $\mathrm{N}$ doses. The interaction of cultivars and years on grain yield indicated that first year (2015/16) is yielding than the second year (2016/17) and Esin variety is yielding than Tacettinbey variety for both years (Fig. 1d).

GGE biplot analysis shows the means over years for nitrogen doses relationships among yield components (Fig. le-h), the results of relationship between $\mathrm{N}$ doses and yield components showed that there is high correlation among components and $\mathrm{N}$ application doses (Fig. 1e). The figures show that there is high correlation between $\mathrm{N}_{2}$ application dose and all yield components, while there is high correlation between $\mathrm{N}_{3}$ doses and GY, LS, NGS, HW TGW and high correlation between $\mathrm{N}_{4}$ and MT, PC, HT, $\mathrm{PH}$ and there is not any correlation among $\mathrm{N}_{0}, \mathrm{~N}_{1}$ and yield components. The sector analysis of $\mathrm{N}$ doses and grouping of traits showed in Fig. If, the results indicated that four sectors occurred on the figure and MT, PC, HT, PH took places in first group in first sector with $\mathrm{N}_{4}$, and GY, LS, NGS, HW TGW took places in second group in second sector with $\mathrm{N}_{3}$, while $\mathrm{N}_{2}$ took places in center of first and second sector, so this dose took places on center of all components. On the other hand $\mathrm{N}_{0}$ and $\mathrm{N}_{1}$ located in different sector and opposite of component group, so did not related with any component. The ranking biplot of $\mathrm{N}$ doses on components showed in Fig. 1 , and the figure indicated that $\mathrm{N}_{2}$ dose is stable for all components, because $\mathrm{N}_{2}$ dose located center of stable line with locate above mean of components line, while $\mathrm{N}_{2}$ doses located side of GY, LS, NGS, HW TGW and desirable for these components, and $\mathrm{N}_{2}$ desirable for MT, PC, HT, PH. On the other hand, $\mathrm{N}_{0}$ and $\mathrm{N}_{1}$ located in under mean of component line, so these doses are undesirable for all components. The comparison biplot of $\mathrm{N}$ doses on components showed in Fig. $1 \mathrm{~h}$, and the figure indicated that $\mathrm{N}_{2}$ dose is stable for all components, because $\mathrm{N} 2$ dose located on ideal enter line, while $\mathrm{N}_{3}$ doses located side of GY, LS, NGS, HW TGW and desirable for these components, and $\mathrm{N}_{4}$ desirable 
for MT, PC, HT, PH. On the other hand, $\mathrm{N}_{0}$ and $\mathrm{N}_{1}$ located in under mean of component line, so these doses are undesirable for all components.

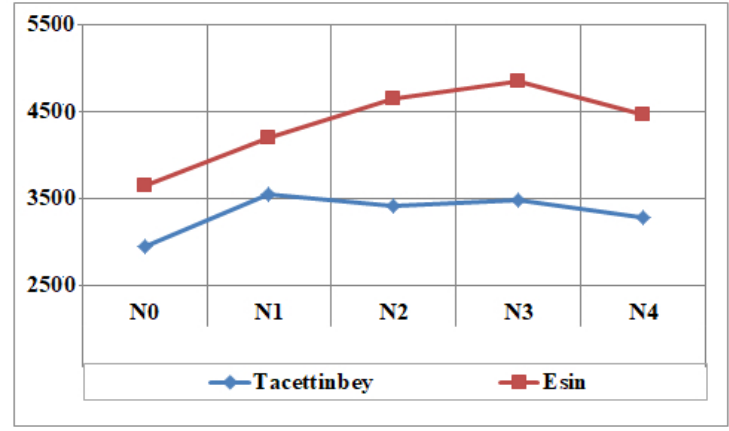

a
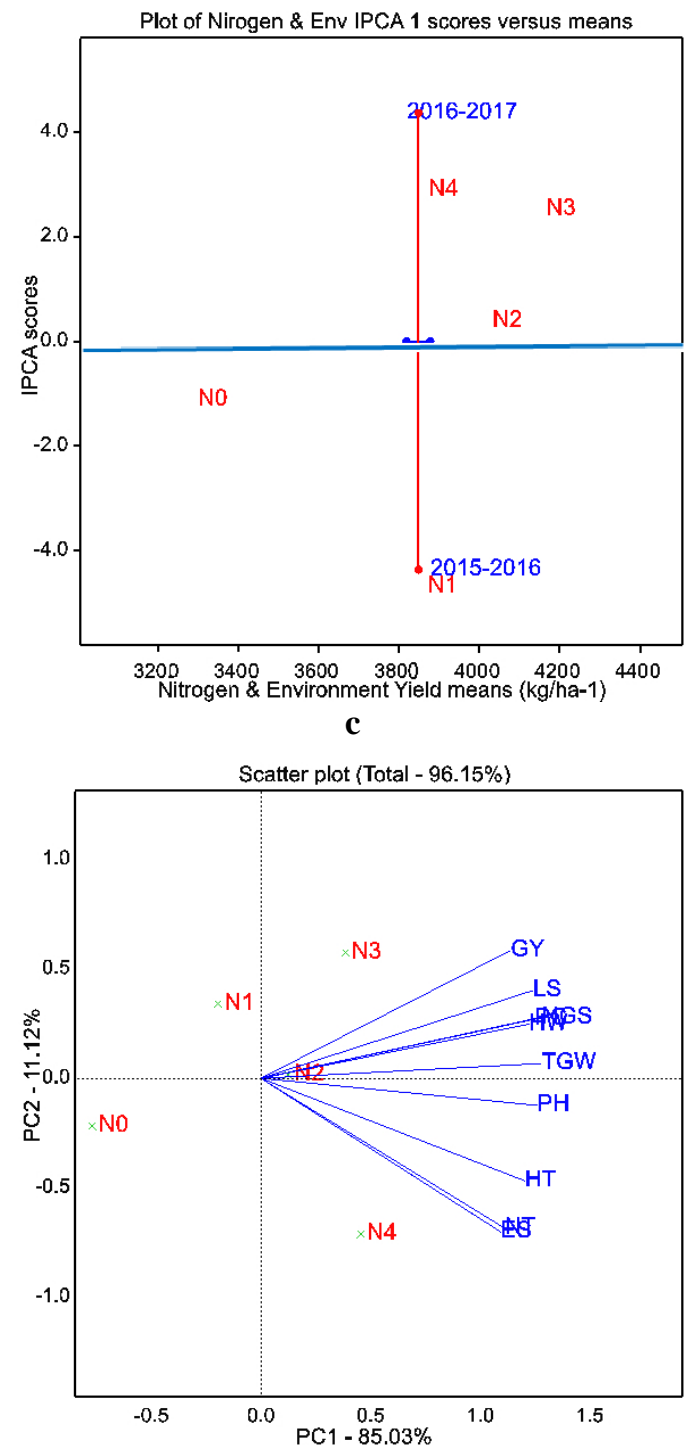

e

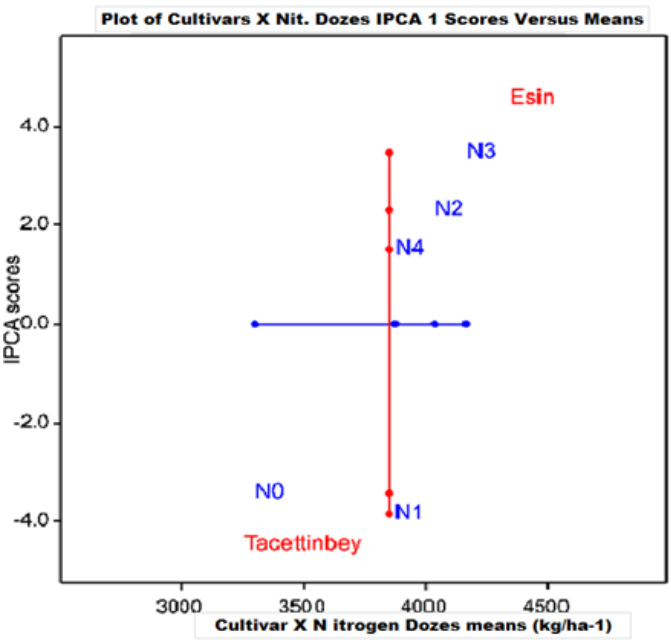

b
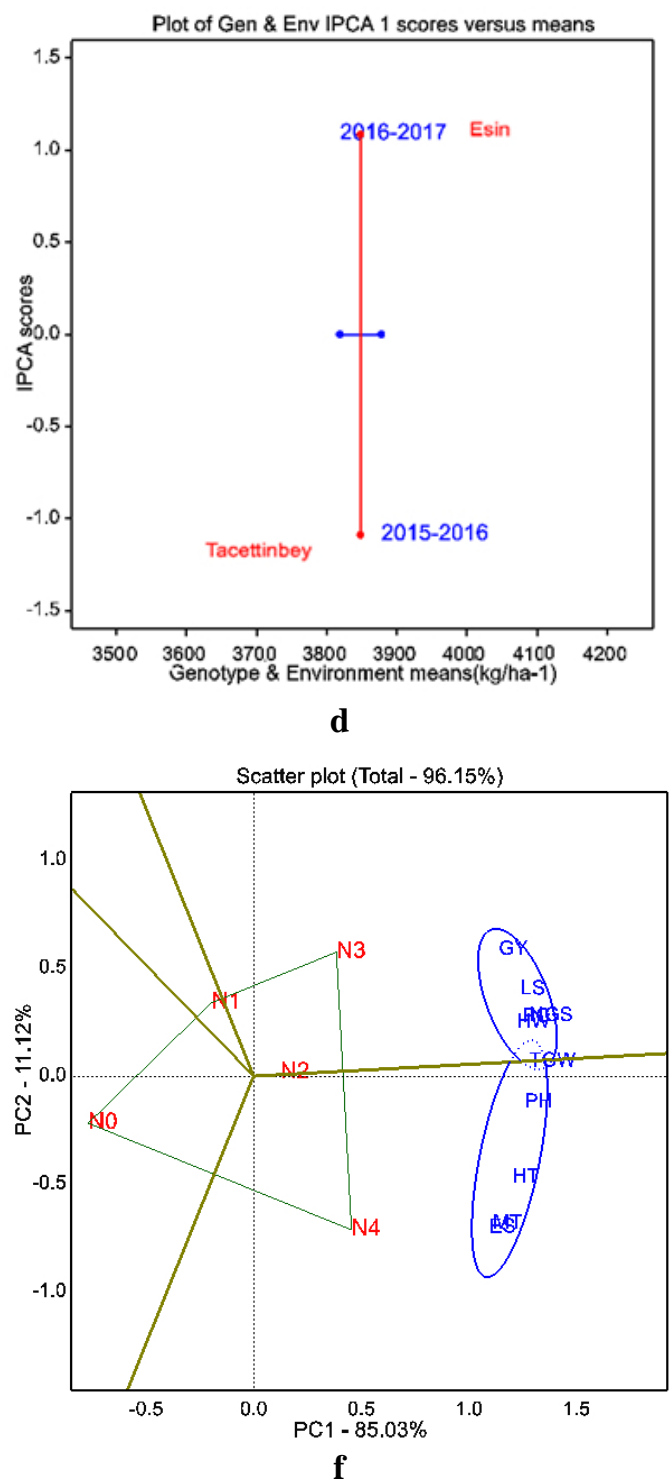

APPLIED ECOLOGY AND ENVIRONMENTAL RESEARCH 16(4):4865-4878. http://www.aloki.hu • ISSN 15891623 (Print) • ISSN 17850037 (Online) DOI: http://dx.doi.org/10.15666/aeer/1604_48654878 

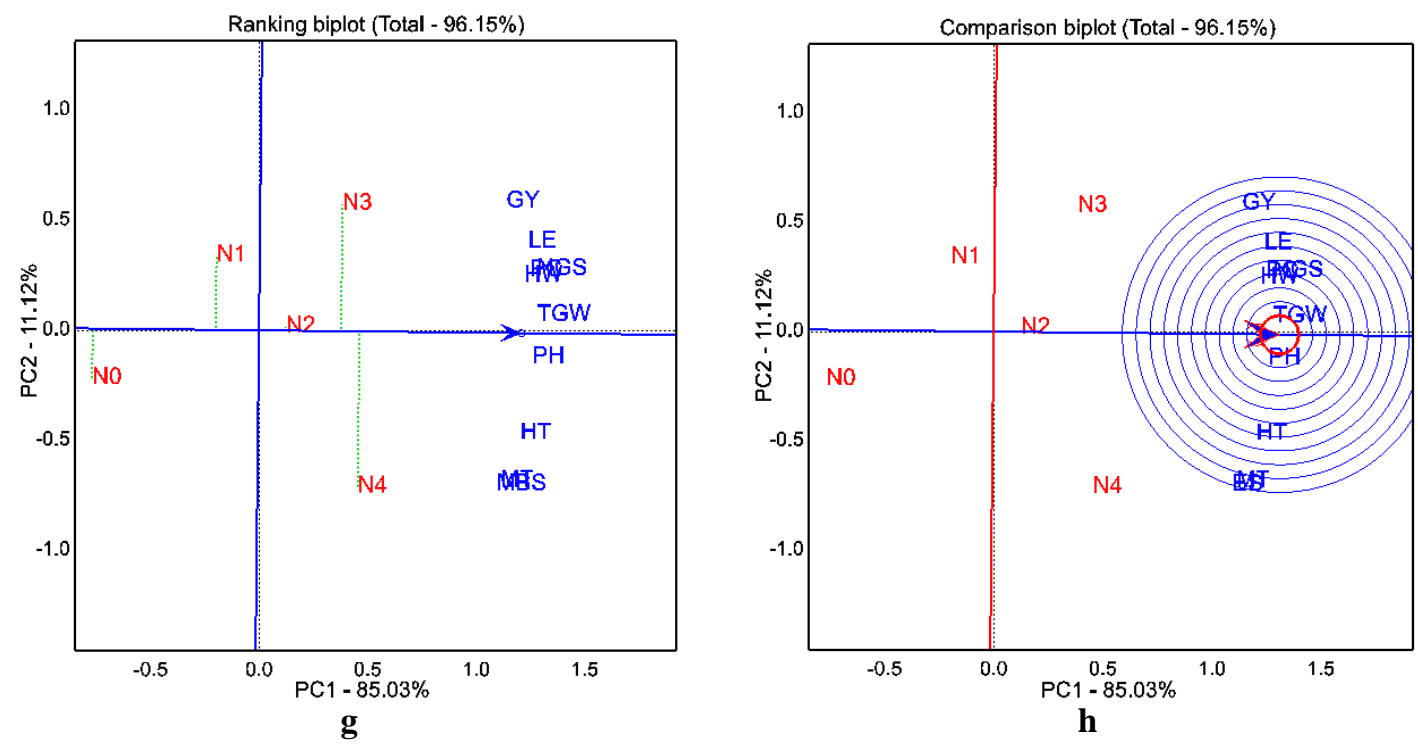

Figure 1. a. Regression analysis of grain yield ( $\mathrm{kg} / \mathrm{ha}$ ) of cultivar and nitrogen doses. b. AMMI analysis of $N$ doses and cultivars on grain yield. $c$. AMMI analysis on grain yield of cultivar. $d$. AMMI analysis of $N$ doses of years. $\boldsymbol{e}$. The relation between $N$ doses and traits. $f$. The sector analysis of $N$ doses and grouping of traits. $g$. Ranking of $N$ doses on traits. $\boldsymbol{h}$. Comparison of $N$ doses on traits

\section{Discussion}

Analysis of variance for grain yield and yield components showed that nitrogen dose, year and cultivar was the main independent factor determining the differences in values of parameters between $\mathrm{N}$ doses and results. The significant differences $(\mathrm{P}<0.001$, $\mathrm{P}<0.005$ ) found of the yield and yield components and the grain yield of triticale was most of all affected by cultivars (70.8\%) and then by nitrogen doses $(23.5 \%)$ and interaction (5.7\%) in Table 5, therefore the effect of nitrogen doses gives a lead to high variable outputs yield and all its components every year. These results are accepted by Moreno et al. (2003), which proceeds that the $\mathrm{N}$ fertilizer highly depends on growing season's variations conditioned by environmental factors. Climatic data of both growing season were indicated that the season of 2015/16 had favorable climate conditions for grain yield, without more cold in winter and good rainfall in planting time (October, November) for early germination and for grain filling time occurs (April, May). On the other hand, the climate conditions for all components without grain yield were good in 2016/17 growing season.

According to results of the study, the application of nitrogen doses had positive effect on yield and yield components. The results increased in parallel with dose increase for both cultivars for all characters. Nitrogen application had positive influence on all the yield components (Fallahi et al., 2008). The best grain yield and majority components results were obtained with $\mathrm{N}_{3}$ nitrogen dosing. Moreover, $\mathrm{N}_{4}$ and $\mathrm{N}_{2}$ nitrogen dosing can be advice for some special components depend on climatic data of seasons. The results of nitrogen use efficiency of the present study agree with the findings of Janušauskaitè (2013) who reported that nitrogen use efficiency for grain yield and protein content as affected by nitrogen rate and its splitting in spring triticale and Yildirim et al. (2007) the findings suggest that advanced breeding lines should be selected at different $\mathrm{N}$ levels for better $\mathrm{N}$ use efficiency and genetic investigations 
should be conducted in multiple environments and Alaru et al. (2009) who reported that yield values are under the influence of application of $\mathrm{N}$ fertilization. There are more $\mathrm{N}$ rate application studies on components in different crop as well as triticale which agree in present study; Hadi et al. (2012), increase in number of time to spike by increasing N rate and it might be attributed to the increase in long time filling grain (Gursoy, 2011; Shafi et al., 2011). Hadi et al. (2012), increase SS m-2 by increasing $\mathrm{N}$ rate and it might be attributed to increase the time of number spike, Hadi et al. (2012), increase in PH by increasing $\mathrm{N}$ rate might be attributed to the increase in vegetative of plant. The increase SL depend on increasing $\mathrm{N}$ rate and available environmental in barley and wheat by researchers (Gursoy, 2011; Shafi et al., 2011). Subhan et al. (2004) and Shafi et al. (2011), increase NGS by increasing $\mathrm{N}$ rate and it might be attributed to increase the time grain occurs. Fallahi et al. (2008) Alazmani (2015), Yildirim et al. (2016), increasing $\mathrm{N}$ rate and it might be attributed to increase the grains per spike, and this is increasing GY.

\section{GGE biplot analysis}

The GGE biplot mainly allows the visualization of any crossover GE interaction, which is very important for the breeding program (Sayar, 2017; Kendal, 2015). The GGE biplot method has been widely used to analyze the stability and performance of the genotypes for yield and other components (Goyal et al., 2011; Tekdal and Kendal, 2017). Moreover, GEI and yield stability analyses are important for their consideration of both varietal stability and suitability for cultivation across seasons and ecological circumstances. The GT (genotype-trait) biplot provides an excellent tool for visualizing genotype $\times$ trait data (Kendal, 2015).

The AMMI analysis shows good visualization of interaction among nitrogen application doses and cultivars (Fig. 1b), among doses and years (Fig. 1c), among years and cultivars (Fig. 1d). The AMMI method provides considerable flexibility, allowing plant breeders to simultaneously select for yield and stability (Kendal and Sener, 2015).

The GGE biplot could be used to interpret the relationships among nitrogen doses, components, and groups of component (Fig. 1e). An understanding of the relationship between doses and components can aid in better understanding doses objectives and in identifying components that are positively or negatively correlated with nitrogen doses. This understanding can also aid in identifying components that can be indirectly selected by selecting for correlated components. It also helps to visualize the strengths and weaknesses of nitrogen doses, which is important for application in barley. If the angle of the vector was less than $90^{\circ}$, there was a positive correlation two observation factors. If the angle was equal to $90^{\circ}$, they were not correlated. There was a negative correlation if the angle was less than $90^{\circ}$ (Yan and Thinker, 2006; Dogan et al., 2107). On the other hand, all components took place in two different groups, with $\mathrm{N}_{2}, \mathrm{~N}_{3}$ and $\mathrm{N} 4$ doses (Fig. If). There is high correlation which is took places in same group (Kendal et al., 2016). Moreover, the GGE biplot was accurate in interpreting the ranking and comparing $\mathrm{N}$ doses and components (Fig. 1g). The doses with both high mean performance and high stability for all of the components were called as table dose $\left(\mathrm{N}_{2}\right)$. The dose located side of some components, it means that this dose can be advise for these components $\left(\mathrm{N}_{3}\right.$ an $\left.\mathrm{N}_{4}\right)$. The center of the concentric circles (i.e., ideal dose) was the AEA in the positive direction. In the comparison biplot doses located closer to the ideal dose were more desirable than others (Yan and Tinker, 2006; Benin et al., 2012; Dogan et al., 2016). The result demonstrated that $\mathrm{N}_{2}$ was ideal application dose in the 
both season for all components, as it was in the center circle for the ideal doses and on the AEA (Fig. 1h).

\section{Conclusions}

1. In the present study nitrogen application doses had positive effect on grain yield and yield components, and the values of component and grain yield increased in parallel with application nitrogen doses.

2. The nitrogen dose of $\mathrm{N} 3\left(120 \mathrm{~kg} \mathrm{ha}^{-1}\right)$ are responsible for the maximum productivity of triticale crop in Mardin province environmental conditions. Esin variety showed that it is best cultivar for all components and grain yield except PC.

3. On the other hand; AMMI and GGE biplot analysis revealed that this analysis provided useful results and high image quality to show the correlation among doses, cultivars and components.

4. The results of study showed that the $\mathrm{N}_{3}$ application of doses in triticale should be recommend for next studies both farm and research area.

\section{REFERENCES}

[1] Alaru, M., Laur, Ü., Eremeev, V., Reintam, E., Selge, A., Noormets, M. (2009): Winter triticale yield formation and quality affected by $\mathrm{N}$ rate, timing and splitting. Agricultural and Food Science 18: 76-90.

[2] Alazmani, A. (2015): Evaluation of yield and yield components of barley varieties to nitrogen. - International Journal of Agriculture and Crop Sciences IJACS 8(1): 52-54.

[3] Anonymous (2002): SAS, Institute. JMP Statistics. - SAS Institute Inc., Cary, NC.

[4] Asfaw, A., Alemayehu, F., Gurum, F., Atnaf, M. (2009): AMMI and SREG GGE biplot analysis for matching varieties onto soybean production environments in Ethiopia. Scientific Res. and Essay 4(11): 1322-1330.

[5] Benin, G., Bornhofen, E., Beche, E., Pagliosa, I. E. S., Lemesda, Silva, C., Pinnow, C. (2012): Agronomic performance of wheat cultivars in response to nitrogen fertilization levels. - Acta Scientiarum, Agronomy Maringá 34(3): 275-283.

[6] Dogan, Y., Kendal, E., Oral, E. (2016): Identifying of relationship between traits and grain yield in spring Barley by GGE biplot analysis. - Agriculture and Forestry 6(24): 239-252.

[7] Estrada-Campuzano, G., Slafer, G. A., Miralles, D. J. (2012): Differences in yield, biomass and their components between triticale and wheat grown under contrasting water and nitrogen environments. - Field Crops Research 128: 167-179. http://dx.doi.org/10.1016/j.fcr.2012.01.003.

[8] Fallahi, H A, Nasseri, A., Siadat, A. (2008): Wheat yield components are positively influenced by nitrogen application under moisture deficit environments. - Int. J. Agri. Biol. 10: 6.

[9] Farshadfar, E., Rashidi, M., Jowkar, M. M., Zali, H. (2013): GGE Biplot analysis of genotype $\times$ environment interaction in chickpea genotypes. - European J. of Exper. Biology 3(1): 417-423.

[10] Goyal, A., Beres, B. L., Randhawa, H. S., Navabi, A., Salmon, D. F., Eudes, F. (2011): Yield stability analysis of broadly adaptive triticale germplasm in southern and central Alberta. - Canadian J. Plant Sci. 91: 125-135.

[11] Gülmezoglu, N., Kinaci, E. (2004): Efficiency of different top dressed nitrogen on triticale ( $\times$ Triticosecale Wittmack) under contrasting precipitation conditions in semiarid 
region. - Pakistan Journal of Biological Sciences 7(3): 353-358. http://dx.doi.org/10.3923/pjbs. 2004. 353.358.

[12] Gürsoy, M. (2011): Effects of different nitrogen doses on yield and quality components in some two-rowed barley (Hordeum vulgare L.) lines and cultivars. - Journal of New World Sciences 6(4): 114-123.

[13] Hadi, F., Arif, M., Hussain, F. (2012): Response of dual purpose barley to rates and methods of nitrogen application. - ARPN Journal of Agricultural and Biological Science 7(7): 533-539.

[14] Janušauskaite, D. (2013): Spring triticale yield formation and nitrogen use efficiency as affected by nitrogen rate and its splitting. - Zemdirbyste-Agriculture 100(4): 383-392. DOI: 10.13080/z-a.2013.100.049.

[15] Kendal, E. (2015): Relationship between chlorophyll and other features in durum wheat (Triticum turgidum L. var. durum) using SPAD and biplot analyses. - Journal of Agricultural Science and Technology 17: 1873-1886.

[16] Kendal, E. (2016): GGE Biplot analysis of multi-environment yield trials in barley (Hordeum vulgare L) cultivars. - Ekin J. of Crop Breeding and Genetics 2(3): 90-99.

[17] Kendal, E., Doğan, Y. (2015): Stability of a candidate and cultivars (Hordeum vulgare L) by GGE biplot analysis of multi-environment yield trial in spring barley. - Agriculture and Forestry/Poljoprivredai Sumarstvo 61: 4.

[18] Kendal, E., Sayar, M. S. (2016): The stability of some spring triticale genotypes using biplot analysis. - The Journal of Animal and Plant Sciences 26(3): 754-765.

[19] Kendal, E., Şener, O. (2015): Examination of genotype $\times$ environment interactions by GGE biplot analysis in spring durum wheat. - Indian J. Genet. 75(3): 341-348.

[20] Kendal, E., Tekdal, S., Aktaş, H., Karaman, M. (2014): Determination compliance abilities of some triticale varieties and comparison with wheat in SoutheasternAnatolia conditions of Turkey. - Communications in Agricultural and Applied Biological Science 79(4): 192-200.

[21] Kendal, E., Sayar, M. S., Tekdal, S, Aktaş, H., Karaman, M. (2016): Assessment of the impact of ecological factors on yield and quality parameters in triticale using GGE biplot and AMMI analysis. - Pak. J. Bot. 48(5): 1903-1913.

[22] Kilic, H. (2014): Additive main effect and multiplicative interactions (AMMI) Analysis of grain yield in barley genotypes across environments. - J. Agr. Sc. 20: 337-344.

[23] Lance, R. Gibson., Carasella, D., Nance, Douglas, L. K. (2007): Winter triticale response to nitrogen fertilization when grown after corn or soybean. - Agron. J. 99: 49-58.

[24] Lestingi, A., Bovera, F., De Giorgio, D., Ventrella, D., Tateo, A. (2010): Effects of tillage and nitrogen fertilization on triticale grain yield, chemical composition and nutritive value. - Journal of the Science of Food and Agriculture 490: 2440-2446. http://dx.doi.org/ 10.1002 /jsfa.410.

[25] Moreno, A., Moreno, M. M., Ribas, F., Cabello, M. C. (2003): Influence of nitrogen fertilizer on grain yield of barley (Hordeum vulgare L.) under irrigated conditions. Spanish Journal of Agricultural Research 1(1): 91-100.

[26] Moselhy, E. I., Zahran, M. A. (2002): Effect of bio and mineral nitrogen fertilization on barley crop grown on a sandy soil. - Egypt. J. Agric. Res. 3: 921-936.

[27] Sayar, M. S. (2017): Additive main effects and multiplicative interactions (AMMI) analysis for fresh forage yield in common vetch (Vicia sativa L.) genotypes. Agriculture and Forestry 63(1): 119-127.

[28] Sayar, M. S., Han, Y. (2015): Determination of seed yield and yield components of grasspea (Lathyrus sativus L.) lines and evaluations using GGE biplot analysis method. TarımBilimleri Der- J. of Agric. Sci. 21(1): 78-92.

[29] Shafi, M, Bakht, J., Jalal, F., Aman, Khan, M., Gül, Khattak, S. (2011): Effect of nitrogen application on yield and yield components of barley (Hordeum vulgare L.). - Pak. J. Bot. 43(3): 1471-1475. 
[30] Steel, G. D., Torrie, J. H. (1980): Principles and Procedures of Statistics: A Biometrical Approach. 2. ed. - McGraw-Hill, New York.

[31] Subhan, W., Anwar, M., Ahmad, N., Gulzar, A., Siddig, M, Rahman, S., Ahmad, I, Rauf, A. (2004): Effect of gamma radiation on growth and yield of barley under different nitrogen levels. - Pakistan Journal of Biological Sci. 7(6): 981-983.

[32] Ugarte, C., Calderini, D. F., Slafer, G. A. (2007): Grain weight and grain number responsiveness to pre-anthesis temperature in wheat, barley and triticale. - Field Crops Research 100: 240-248. http://dx.doi.org/10.1016/j.fcr.2006.07.010.

[33] Yan, W., Tinker, N. A. (2006): Biplot analysis of multi-environment trial data. Principles and applications. - Canadian Journal of Plant Science 86: 623-645.

[34] Yildirim, M., Yakut, Z., Akınc1, C., Kurt, F., Kizilgeci, F. (2016): Nitrogen rate and timing implementation on durum wheat in a bed planting system. - Sains Malaysiana 45(2): 221-228.

[35] Yildirim, M., Bahar, B., Genc, I., Korkmaz, K., Karnez, E. (2007): Diallel analysis of wheat parents and their F2 progenies under medium and low level of available $\mathrm{n}$ in soil. Journal of Plant Nutrition 30: 937-945. 